\title{
Evidências de validade da Escala Brasileira de Solidão UCLA
}

\author{
Evidence of validity of Brazilian UCLA Loneliness Scale
}

Sabrina Martins Barroso', Valéria Sousa de Andrade², Aida Hutz Midgett ${ }^{3}$, Rubem Gomes Neves de Carvalho ${ }^{1}$

\section{RESUMO}

Objetivo: Este trabalho investigou as evidências de validade da Escala de Solidão UCLA para aplicação na população brasileira. Métodos: Foram seguidas as fases: (1) autorização do autor e do Comitê de Ética; (2) tradução e retrotradução; (3) adaptação semântica; (4) validação. Utilizou-se para análise dos dados análise descritiva, fatorial exploratória, alpha de Cronbach, Kappa, teste de esfericidade de Barlett, teste Kaiser-Meyer-Olkin e correlação de Pearson. Para a adaptação, a escala foi submetida a especialistas e a um grupo focal com 8 participantes para adaptação semântica e a um estudo piloto com 126 participantes para adaptação transcultural. Da validação, participaram 818 pessoas, entre 20 e 87 anos, que responderam a duas versões da UCLA, ao Questionário de Saúde do Paciente, à Escala de Percepção de Suporte Social e a um questionário elaborado pelos autores. Resultados: A escala mostrou dois fatores, que explicaram $56 \%$ da variância e alpha de 0,94. Conclusões:

\section{Palavras-chave}

Psicometria, validade dos testes, solidão, questionários. A Escala de Solidão UCLA-BR indicou evidências de validade de construto e discriminante, além de boa fidedignidade, podendo ser utilizada para avaliação da solidão na população brasileira.

\section{Keywords}

Psychometrics, validity

of tests, loneliness, questionnaires.

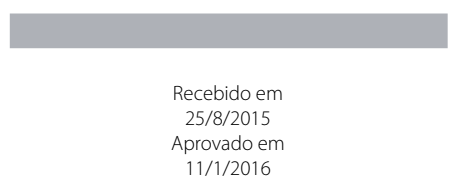

DOI: $10.1590 / 0047-2085000000105$

\section{ABSTRACT}

Objective: This study investigated the validity evidence of Loneliness Scale UCLA to be used in the Brazilian population. Methods: It was followed the phases authorization of the author and the Ethics Committee, translation and back-translation, semantic adaptation and validation of the test. Data were analyzed by descriptive analysis, exploratory factor, alpha Chronbach, Kappa, sphericity test of Bartlett, Kaiser-Meyer-Olkin test and Pearson's correlation. To semantic adaptation the scale was submitted to specialists and a focus group with 8 participants and to cross-cultural adaptation the scale was submitted to a pilot study with 126 participants. In the validation participated 818 people, between 20 and 87 years, who responded to two versions of UCLA, Patient Health Questionnaire, Social Support Scale and a questionnaire made by the authors. Results: The scale showed two factors, which explained $56 \%$ of variance and alpha of 0.94 . Conclusions: Loneliness Scale UCLA-BR indicated evidence of discriminant and construct validity, as well as good reliability. It can be used for evaluation of loneliness in the Brazilian population.

1 Universidade Federal do Triângulo Mineiro (UFTM), Departamento de Psicologia. 2 UFTM, Departamento de Terapia Ocupacional.

3 Boise University, Department of Counselor Education, Boise, Idaho, USA.

Endereço para correspondência: Sabrina Martins Barroso Rua Carmelita Rezende, 70, bloco F, ap. 03, Parque do Mirante 38081-480 - Uberaba, MG, Brasil Telefones: (34) 9917-0850/(34) 3317-4260

E-mail: smb.uftm@gmail.com 


\section{INTRODUÇÃO}

A solidão é uma experiência comum à espécie humana. Alguns estudos internacionais indicam que $80 \%$ das pessoas abaixo de 18 anos e 40\% das acima de 65 anos relatam sentir solidão pelo menos algumas vezes por semana ${ }^{1,2}$. Um estudo sobre a prevalência da solidão entre idosos em 12 países diferentes ${ }^{3}$ mostrou variação na prevalência da solidão entre 25\% na Dinamarca a 60\% na Grécia. No Brasil essa informação ainda não foi estimada.

Além disso, estudos sobre a população norte-americana mostram que entre 15\% e 30\% das pessoas acima de 65 anos sentem-se cronicamente solitárias ${ }^{4,5}$. Esses são resultados preocupantes, pois a solidão tem sido associada com maior risco para o desenvolvimento de transtornos mentais ${ }^{6-8}$, em especial a depressão 9 . A primeira versão da Escala de Solidão UCLA já demonstrava correlação moderada com a triagem para depressão em $1978^{10}$, tendo mostrado melhora nessa relação com sua versão revisada"1.

Outros aspectos negativos relacionados com a solidão são as piores condições físicas ${ }^{8}$, o aumento de processos inflamatórios ${ }^{12}$, o aumento do declínio cognitivo e surgimento de demências ${ }^{13}$ e maior risco de autoextermínio ${ }^{9,14}$. Essas associações têm sido observadas em várias faixas etárias ${ }^{13-15}$. A população acima de 60 anos tem sido a mais investigada, pois essa é uma fase da vida em que há maiores limitações físicas e perda de suporte social| ${ }^{15-17}$, tornando as possíveis consequências da solidão mais intensas.

Não há uma definição consensual para solidão, havendo indícios de que ela pode se manifestar de formas distintas entre crianças/adolescentes, adultos e idosos ${ }^{18,19}$. Um ponto de concordância entre os autores que investigam o conceito é que não ter outras pessoas por perto, ou seja, estar isolado socialmente, não é sinônimo de solidão $0^{4,20}$. Uma das definições mais adotadas para a compreensão da solidão, que embasa boa parte dos instrumentos que mensuram a solidão de adultos e idosos, a define como um conceito bifatorial, conhecido como "modelo de discrepância cognitiva", entendendo solidão como uma percepção cognitiva de que os relacionamentos sociais existentes na vida são insuficientes ou inadequados, gerando uma reação afetiva de tristeza e vazio ${ }^{4,20,21}$. Assim, a solidão tem um componente cognitivo, de interpretação de uma situação, e um componente emocional, derivado dessa percepção. A solidão não depende de estar objetivamente sozinho, pois caso não haja sentimentos negativos associados ao fato de estar sozinho, essa condição não representa solidão. De forma equivalente, mesmo estando cercado por diversas pessoas, alguém pode se sentir solitário.

Essa diferença entre o aspecto objetivo e a percepção de ter alguém com quem contar reflete a relação entre suporte social e solidão. Como o suporte social é baseado na percepção subjetiva de ter alguém com quem se pode contar e a quem se pode procurar em momentos de necessidade, os estudos têm mostrado uma relação negativa entre tal conceito e a solidão $0^{20,22,23}$. Pessoas que se percebem amadas e protegidas, mesmo que estejam distantes de seus familiares e amigos, não se sentem solitárias ${ }^{15,20}$.

Internacionalmente há vários instrumentos disponíveis para avaliação da solidão, tais como a Loneliness and Aloneness Scale for Children and Adolescents ${ }^{24}$, a Three-Item Loneliness Scale ${ }^{25}$ e as diferentes versões da UCLA Loneliness Scale ${ }^{10,11,26}$. Sendo a UCLA Loneliness Scale (Escala de Solidão UCLA), em suas várias versões, uma das mais utilizadas ${ }^{27-29}$.

No Brasil houve um estudo inicial sobre as qualidades psicométricas da primeira versão da Escala de Solidão UCLA, em meados da década de $1980^{30}$, mas, além de o tempo de publicação desse trabalho ser superior ao indicado pelo Conselho Federal de Psicologia (CFP), como o recomendado para manter um instrumento aconselhável para uso pelos profissionais no Brasil| ${ }^{31}$, o trabalho contava com amostragem restrita, apenas 290 pessoas, todas estudantes universitárias.

Considerando os possíveis impactos da solidão no surgimento ou agravamento de condições físicas e emocionais e a boa aceitação da Escala de Solidão UCLA em contexto internacional, o presente trabalho visou adaptar para o contexto brasileiro e investigar evidências de validade e fidedignidade nesse instrumento.

\section{MÉTODOS}

Trata-se de um estudo metodológico descritivo cujos participantes consistiram de pessoas de ambos os sexos, com idade entre 20 e 87 anos, residentes nos estados de Minas Gerais, São Paulo, Espírito Santo, Rio Grande do Sul, Goiás e Tocantins, totalizando 944 participantes (Tabela 1). Essa amostra foi dividida entre as diferentes etapas do estudo, como indicado:

- Etapa de adaptação semântica: oito participantes, com idade entre 20 e 70 anos e escolaridade entre primeiro grau incompleto e terceiro grau completo. Esses participantes foram escolhidos por conveniência e recrutados entre os contatos sociais da equipe de pesquisa, visando garantir variabilidade dos respondentes dentro da população de interesse para o estudo.

- Etapa de adaptação transcultural: 116 participantes, com idades entre 20 e 87 anos e escolaridade entre primeiro grau incompleto e terceiro grau completo. Esses participantes foram escolhidos por conveniência e recrutados entre estudantes universitários, familiares e amigos dos estudantes, idosos comunitários frequentadores da Unidade de Atenção do Idoso (UAI) e idosos institucionalizados em Instituições de Longa Permanência para Idosos de Uberaba, sem diagnóstico de patologias psiquiátricas. 
- Etapa de validação: o cálculo amostral foi feito considerando indicação da literatura de haver um mínimo de 10 pessoas para cada item do instrumento original que se deseja validar ${ }^{32}$, acrescido da recomendação de incluir uma porcentagem de $20 \%$ a $30 \%$ referente a possíveis perdas ${ }^{33}$. Assim, definiu-se a amostra mínima do presente estudo em 300 participantes.

Procedeu-se, então, a um sorteio aleatório estratificado, com reposição, com base na lista telefônica da cidade de Uberaba, listagem de frequentadores da Universidade Aberta da Terceira Idade da cidade, frequentadores da UAI e das nove instituições de longa permanência para idosos cadastradas na Secretaria Municipal de Saúde de Uberaba. O sorteio para composição da amostra respeitou a proporção mínima de pessoas acima de 60 anos da cidade, de 12,6\% segundo o censo de $2010^{34}$, e o critério de exclusão de descartar possíveis participantes com menos de 20 anos. Essa decisão foi tomada porque nenhuma versão da Escala de Solidão UCLA original foi desenvolvida para aplicação em crianças e adolescentes. Todos os sorteados foram contatados por telefone ou pessoalmente e participaram do estudo apenas os que assinaram o termo de consentimento livre e esclarecido.

Tabela 1. Caracterização da amostra, $2014(\mathrm{~N}=944)$

\begin{tabular}{|c|c|c|c|c|}
\hline & \multicolumn{2}{|c|}{ Adaptação } & \multicolumn{2}{|c|}{ Validação } \\
\hline & $\mathrm{n}$ & $\%$ & $\mathrm{n}$ & $\%$ \\
\hline \multicolumn{5}{|l|}{ Sexo } \\
\hline Feminino & 106 & 66,00 & 534 & 63,70 \\
\hline Masculino & 54 & 34,00 & 304 & 36,30 \\
\hline \multicolumn{5}{|l|}{ Escolaridade } \\
\hline Fundamental incompleto & 17 & 10,60 & 17 & 2,00 \\
\hline Fundamental completo & 31 & 19,10 & 16 & 1,90 \\
\hline Médio incompleto & 03 & 2,10 & 35 & 4,20 \\
\hline Médio completo & 24 & 14,90 & 91 & 10,90 \\
\hline Superior incompleto & 54 & 34,00 & 287 & 34,20 \\
\hline Superior completo & 24 & 14,90 & 385 & 45,90 \\
\hline Não informado & 07 & 4,30 & 07 & 0,80 \\
\hline \multicolumn{5}{|l|}{ Estado civil } \\
\hline Solteiro & 68 & 42,60 & 591 & 70,50 \\
\hline Casado & 68 & 42,60 & 183 & 21,80 \\
\hline Separado/divorciado & 07 & 4,30 & 61 & 7,30 \\
\hline Viúvo & 17 & 10,60 & 02 & 0,20 \\
\hline \multicolumn{5}{|l|}{ Considera-se solitário } \\
\hline Nenhum pouco & & & 125 & 14,90 \\
\hline Às vezes & & & 391 & 46,70 \\
\hline Frequentemente & & & 134 & 16,00 \\
\hline Sempre & & & 47 & 5,60 \\
\hline Não informado & & & 141 & 16,80 \\
\hline
\end{tabular}

Após completar o total mínimo para investigação das propriedades psicométricas da versão brasileira da Escala de Solidão UCLA ( $n=300)$, abriu-se uma etapa de coleta online, para ampliação da variabilidade amostral. Essa etapa da coleta ocorreu via internet, por meio do site SurveyMonkey, onde se obtiveram respostas de 586 participantes. Precisou-se, entretanto, eliminar as respostas incompletas ou de menores de 20 anos, finalizando a coleta online em 518 respondentes, de diversas cidades de Minas Gerais, São Paulo e algumas cidades do Espírito Santo, Rio Grande do Sul, Goiás e Tocantins.

\section{Instrumentos}

Duas versões da Escala de Solidão. Escala de Solidão UCLA Revisada (R-UCLA), desenvolvida por Russel et al. ${ }^{11}$. Essa versão revisada da Escala de Solidão UCLA contém 20 afirmações e apresenta as alternativas de resposta em forma de escala likert de quatro pontos, indo de 1 (nunca) a 4 (frequentemente). Essa escala apresentou boa consistência interna $(a=0,94)$, correlação com a autopercepção sobre a solidão $(r=0,70)$ e validade discriminativa com diversos outros conceitos, entre eles a depressão $(r=0,50)$, autoestima $(r=-0,49)$, introversão/extroversão $(r=-0,46)$, tendência à afiliação $(r=$ $-0,45)$, ansiedade $(r=0,36)$, assertividade $(r=-0,34)$, sensibilidade à rejeição $(r=0,27)$ e desejabilidade social $(r=-0,20)$.

Escala de Solidão UCLA 3, desenvolvida por Russe ${ }^{26}$. Apresenta 20 perguntas relacionadas à frequência com que o respondente vivenciou situações de convívio social e atividades desenvolvidas isoladamente. As alternativas de resposta estão em escala likert de quatro itens, variando entre 1 (nunca) e 4 (sempre). Essa versão apresentou coeficiente de consistência interna entre 0,89 e 0,94, dependendo da amostra do estudo, correlação teste-reteste de 0,73 após um ano, correlação com outros instrumentos que também avaliavam a solidão, incluindo da UCLA-R e escalas de depressão ( $r=$ $0,52)$, desejabilidade social $(r=-0,21)$, suporte social $(r=-0,48$ a -0,56), autoestima $(r=0,60)$ e o Inventário de Personalidade de Eysenck $(r=-0,40$ a 0,49). Nenhuma das duas versões possui ponto de corte e a solidão é avaliada como mais intensa à medida que a pontuação é maior na soma total das respostas aos itens.

Questionário de Saúde do Paciente (PHQ-9). Validada para a população ambulatorial do Brasil $^{35}$ e para a população geral ${ }^{36}$, esta escala faz a triagem para Episódio Depressivo Maior por meio de nove afirmações, respondidas em forma de escala likert de quatro pontos. Esse instrumento encontra-se validado para a população entre 16 e 88 anos e demonstrou correlação com a Entrevista Diagnóstica Internacional Composta (CIDI) de 0,9735. Os itens avaliam a frequência dos sintomas depressivos nos últimos 15 dias, variando de 0 (nenhuma vez) a 3 (quase todos os dias). A pontuação máxima da PHQ-9 é de 27 pontos, indicando: ausência de depressão (0-9 pontos), depressão leve (10-14 pontos), depressão moderada (15-19 pontos) e depressão severa (20-27 pontos). 
Escala de Percepção de Suporte Social. Instrumento validado ${ }^{37}$, composto por 29 itens que avaliam a percepção de existência de suporte social prático e emocional na vida do respondente. As respostas são apresentadas em escala likert de quatro pontos, indo de 1 (nunca) a 4 (sempre). A escala oferece um escore total, um escore de suporte social prático e um emocional. Não apresenta ponto de corte, e valores maiores indicam a presença de melhor rede de apoio social.

Questionário estruturado. Especialmente construído para esse estudo, incluiu questões sociodemográficas e uma pergunta sobre a percepção subjetiva da solidão, elaborada como "Você se considera uma pessoa solitária?", e contava com quatro opções de resposta, em escala tipo likert, indo de "nenhum pouco" até "sempre".

\section{Procedimentos}

O presente estudo foi composto por quatro etapas, seguindo indicação ${ }^{32}$ : (1) Obtenção de autorização e aprovação por Comitê de Ética em Pesquisa; (2) Tradução e retrotradução; (3) Adaptação semântica e cultural; (4) Validação.

\section{Coleta de dados}

Etapa 1: Em fevereiro de 2013 foi obtida autorização do autor da Escala de Solidão UCLA, professor Daniel W. Russel, para sua validação para o Brasil. Após essa autorização, o projeto foi encaminhado e aprovado por um Comitê de Ética em Pesquisa (Protocolo no 2664/2013).

Etapa 2: A tradução e a retrotradução foram feitas por dois profissionais da área de saúde, com fluência em inglês e português, sendo um deles um cidadão norte-americano e o outro brasileiro. As traduções e retrotraduções foram feitas pelos mesmos profissionais em virtude da necessidade de que os envolvidos entendessem dos construtos do instrumento e dos aspectos culturais em que o instrumento foi criado e no que seria utilizado. No entanto, para minimizar falhas de tradução ou retrotradução por vício de linguagem ou parcialidade dos profissionais, todas as versões de tradução e retrotradução foram submetidas à avaliação de um terceiro profissional de saúde, também fluente em inglês e português, não envolvido diretamente nas etapas de tradução e retrotradução, que havia feito a passagem dos instrumentos do inglês para o português de forma independente. Foram feitas traduções da UCLA-R e UCLA 3 de forma independente. Todas as versões foram comparadas pela equipe da pesquisa e as discordâncias foram discutidas por e-mail entre os tradutores, em seguida foram retrotraduzidas e comparadas, até que se chegou à primeira versão em português para cada Escala de Solidão UCLA (UCLA-R e UCLA 3).

Etapa 3: As versões em português da escala UCLA-R e UCLA 3 foram submetidas à avaliação por dois profissionais de saúde com experiência de mais de cinco anos em trabaIhar com avaliação emocional, depressão, suporte social e qualidade de vida, para análise da adequação do conteúdo das escalas para investigação da solidão em contexto brasileiro. Os profissionais consideraram as duas versões adequadas.

Na sequência, a UCLA-R e a UCLA 3 foram submetidas a um grupo focal para verificação de sua compreensão pela população de interesse do instrumento. O grupo focal foi audiogravado para permitir análise posterior e conduzido de forma que, inicialmente, todos os participantes responderam às duas versões traduzidas de maneira independente. Somente depois de os participantes terem respondido aos dois instrumentos, um integrante da equipe de pesquisa leu cada um dos itens em voz alta e tais itens foram discutidos pelos participantes. Buscou-se identificar o que os respondentes entenderam sobre o que cada questão perguntava, a adequação dos termos e possíveis problemas de interpretação ou duplicidade de entendimento.

Foram feitas alterações nos instrumentos segundo as respostas do grupo focal e, posteriormente, as duas escalas foram submetidas a um estudo piloto. Nessa etapa buscou-se fazer uma análise preliminar de consistência interna das versões da Escala de Solidão UCLA por meio de análise de alpha de Cronbach e análise fatorial exploratória, com foco nos componentes principais, utilizando-se rotação Varimax. O nível de concordância entre os resultados das duas versões das escalas foi avaliado por meio de correlação (correlação de Pearson) e do índice Kappa. A versão que mostrou melhores resultados no estudo piloto (UCLA-R) foi enviada ao autor para avaliação da tradução e não recebeu alterações, sendo considerada adaptada para o contexto brasileiro.

Etapa 4: Só então a UCLA-R, agora nomeada como Escala Brasileira de Solidão UCLA (UCLA-BR), foi submetida a estudos sobre evidências de validade. A validade de construto foi verificada por meio da análise do alpha de Cronbach, análise fatorial exploratória, com foco nos componentes principais e rotação Varimax, teste de esfericidade de Barlett e do teste Kaiser-Meyer-Olkin e correlação com a autopercepção da solidão. Enquanto a validade discriminante foi investigada por meio da análise correlacional (correlação de Pearson) entre a UCLA-BR e dois construtos relacionados à solidão em estudos prévios da escala original e posteriores - suporte social e depressão.

A fidedignidade da UCLA-BR foi avaliada por sua consistência interna e também pela correlação do escore total do instrumento com a percepção subjetiva de solidão indicada pelos respondentes do estudo ao responder a questão "Você se considera uma pessoa solitária?".

\section{RESULTADOS}

\section{Adaptação}

A avaliação dos profissionais de saúde considerou as duas versões da Escala de Solidão avaliadas (UCLA-R e UCLA 3) 
como pertinentes para a mensuração da solidão. Durante o grupo focal os participantes indicaram termos que não entenderam, tais como "sente que não está mais próximo de ninguém", "sinto que alguém é amigável" e a apresentação das respostas, que era codificada apenas com as primeiras letras das palavras no original e explicadas em uma legenda apresentada como nota de rodapé na escala. Soluções foram propostas e as consideradas aceitáveis pelo grupo foram incorporadas aos instrumentos.

Durante a etapa de estudo piloto, a UCLA-R apresentou boa consistência interna $(a=0,90)$ e indicou a presença de dois fatores, que explicaram $64,80 \%$ da variância das respostas. A UCLA 3 apresentou consistência interna insatisfatória $(a=0,35)$ e sua análise fatorial suportou até cinco fatores para explicar 62,35\% da variância das respostas.

A análise de correlação entre os resultados das duas versões da escala mostrou correlação baixa entre os instrumentos $(r=0,17 ; p \leq 0,005)$ e o índice Kappa mostrou haver concordância pobre entre eles (Kappa $=0,01 ; p=0,754$ ).

Diante desses resultados, optou-se por seguir com a validação apenas da UCLA-R, passando a nomeá-la como UCLA-BR (Anexo 1). A cotação da escala deve ser feita atribuindo pontuação zero para todas as respostas "nunca", pontuação 1 para as respostas "raramente", pontuação 2 para as respostas "algumas vezes" e pontuação 3 para as respostas "frequentemente". A escala ainda não possui ponto de corte estabelecido, devendo-se considerar que as pessoas com maior pontuação se encontram mais solitárias.

\section{Evidências de validade}

A UCLA-BR mostrou boa consistência interna $(a=0,94)$. A análise fatorial indicou a presença de dois fatores, que explicaram 56\% da variação dos dados (36,95\% para fator 1 e $19,03 \%$ para fator 2 ). As cargas fatoriais variaram entre 0,03 e 0,80 (Tabela 2). A representação gráfica das análises fatoriais encontra-se apresentada na Figura 1.

A correlação intraclasse variou entre 0,93 e 0,95 $(p<0,001)$ e a correlação item-total variou de 0,42 a 0,76 $(p<0,001)$. A adequação desse modelo foi verificada por meio do teste de esfericidade de Bartlett $\left(X^{2}=9268,42 ; p<0,001\right)$ e do teste Kaiser-Meyer-Olkin ( $\mathrm{KMO}=0,96 ; p<0,001)$. A fidedignidade da UCLA-BR foi avaliada por sua consistência interna e também pela expressiva correlação com a percepção subjetiva de solidão dos respondentes $(r=67 ; p<0,001)$.

Tabela 2. Análise fatorial da Escala Brasileira de Solidão UCLA (UCLA-BR), $2014(n=818)$

\begin{tabular}{|c|c|c|c|c|}
\hline \multirow{2}{*}{ Itens } & \multirow{2}{*}{ Correlação item-total } & \multicolumn{2}{|c|}{ Análise fatorial } & \multirow{2}{*}{ Comunalidade } \\
\hline & & $\mathrm{F} \mathbf{1}^{1}$ & $\mathrm{~F}^{2}$ & \\
\hline 1. Eu me sinto infeliz por fazer tantas coisas sozinho(a). & 0,60 & 0,64 & 0,39 & 0,56 \\
\hline 2. Eu não tolero ficar tão sozinho(a). & 0,42 & 0,45 & 0,66 & 0,63 \\
\hline 3. Eu sinto que não tenho companhia. & 0,68 & 0,71 & 0,04 & 0,51 \\
\hline 4. Eu sinto que ninguém me compreende. & 0,65 & 0,69 & $-0,04$ & 0,47 \\
\hline 5. Eu fico esperando as pessoas me ligarem ou escreverem. & 0,57 & 0,61 & 0,26 & 0,45 \\
\hline 6. Eu sinto que não tenho ninguém a quem eu possa recorrer. & 0,64 & 0,69 & $-0,05$ & 0,47 \\
\hline 7. Eu não me sinto próximo(a) a ninguém. & 0,67 & 0,72 & $-0,20$ & 0,55 \\
\hline 8. Sinto que meus interesses e ideias não são compartilhados por aqueles que me rodeiam. & 0,59 & 0,64 & $-0,30$ & 0,49 \\
\hline 9. Eu me sinto excluído(a). & 0,75 & 0,79 & $-0,11$ & 0,63 \\
\hline 10. Eu me sinto completamente sozinho(a). & 0,76 & 0,80 & $-0,04$ & 0,63 \\
\hline 11. Eu sou incapaz de me aproximar e de me comunicar com as pessoas ao meu redor. & 0,61 & 0,66 & $-0,15$ & 0,46 \\
\hline 12. Eu sinto que minhas relações sociais são superficiais. & 0,59 & 0,64 & $-0,23$ & 0,46 \\
\hline 13. Eu me sinto carente de companhia. & 0,68 & 0,72 & 0,28 & 0,59 \\
\hline 14. Eu sinto que ninguém me conhece realmente bem. & 0,62 & 0,66 & $-0,21$ & 0,48 \\
\hline 15. Eu me sinto isolado(a) das outras pessoas. & 0,78 & 0,82 & $-0,19$ & 0,71 \\
\hline 16. Sou infeliz estando tão excluído(a). & 0,74 & 0,77 & 0,18 & 0,63 \\
\hline 17. Para mim é difícil fazer amigos. & 0,64 & 0,69 & $-0,26$ & 0,54 \\
\hline 18. Eu me sinto bloqueado(a) e excluído(a) por outras pessoas. & 0,75 & 0,79 & $-0,07$ & 0,63 \\
\hline 19. Sinto que as pessoas estão ao meu redor, mas não estão comigo. & 0,73 & 0,77 & $-0,14$ & 0,61 \\
\hline 20. Eu me sinto incomodado(a) em realizar atividades sozinho(a). & 0,50 & 0,53 & 0,61 & 0,66 \\
\hline Explicação da Variância & & 36,95 & 19,03 & \\
\hline
\end{tabular}

${ }^{1}$ Carga fatorial do item no fator $1 ;{ }^{2}$ Carga fatorial do item no fator 2. 


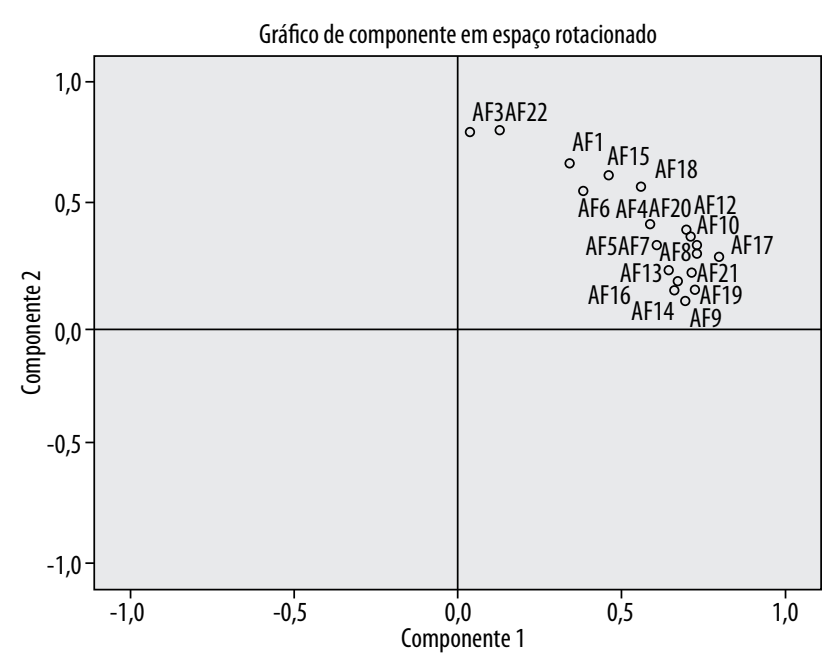

Figura 1. Representação gráfica do agrupamento fatorial da Escala UCLA-BR.

Verificou-se, também, a capacidade discriminante da UCLA-BR, com relação à intensidade de sintomas depressivos e à percepção de suporte social. Essa análise mostrou correlação positiva entre a solidão e a triagem positiva para depressão $(r=0,59 ; p<0,001)$ e correlação negativa com o suporte social $(r=-0,34 ; p<0,001)$, bem como os dois aspectos avaliados do suporte social, o aspecto prático $(r=-0,34$; $p<0,001)$ e o suporte emocional $(r=-0,58 ; p<0,001)$.

\section{DISCUSSÃO}

A solidão, por se tratar de uma percepção subjetiva, aumenta a necessidade de sistematização dos instrumentos utilizados para mensurá-la de forma a garantir que o clínico ou pesquisador tenha uma mínima compreensão sobre o que a pessoa avaliada está vivenciando e nomeando como solidão. Nesse sentido, o presente trabalho adotou a definição de solidão proposta no Modelo de Discrepância Cognitiva, que a considera como uma percepção cognitiva e emocional da discrepância entre o contato social desejado e o vivenciado ${ }^{4,20,27,38}$. Os resultados da análise fatorial e da consistência interna indicaram a adequação da UCLA-BR a esse entendimento da solidão, fornecendo evidências de validade de construto.

A UCLA-R havia mostrado a de Cronbach de 0,94 e correlação moderada com a medida de depressão. A UCLA-BR também evidenciou a de Cronbach de 0,94 e manteve a correlação com a medida de depressão, ainda que a escala correlacionada tenha sido diferente. Esses resultados demonstram que a UCLA-BR manteve as boas qualidades psicométricas da escala original, podendo ser utilizada para mensuração da solidão.

Ter instrumentos capazes de mensurar a solidão tem sido reconhecido como um aspecto importante entre pro- fissionais de saúde mental e pesquisadores, pois possibilita a comparação entre diferentes estudos ${ }^{13,14,17}$, bem como possibilita identificar um fator associado a diversas patologias físicas e psiquiátricas $89,13,14$.

Os resultados demonstraram a capacidade discriminativa da UCLA-BR com relação a dois construtos que a literatura frequentemente associa à solidão: a depressão e o suporte social. Observou-se correlação positiva moderada com a depressão e negativa moderada com a percepção de suporte social. A correlação negativa entre a pontuação na escala de solidão e na escala de suporte social é esperada pela literatura sobre os dois conceitos, 15,19,22,23,27. De forma similar, a associação entre a depressão e a solidão vem sendo demonstrada internacionalmente em diversos estudos ${ }^{9,22}$.

A demonstração dessas capacidades da UCLA-BR, somada com a correlação da pontuação do instrumento com a autopercepção dos respondentes sobre sua solidão $(r=0,67)$, demonstra que a UCLA-BR pode ser uma forma útil de mensurar a solidão na população brasileira. A validação desse instrumento permitirá, entre outras coisas, estimar a prevalência da solidão na população brasileira acima de 20 anos.

O estudo apresenta algumas limitações que precisam ser indicadas. A escala avaliada é de autorrelato e remete a um construto emocional, podendo sua percepção ser afetada por distorções cognitivas relacionadas à memória e motivação para responder. Além disso, não houve investigação da capacidade de estabilidade temporal da medida, de diferenças por faixas etárias ou gênero, ou definição de ponto de corte a partir do qual o instrumento seja mais discriminativo para solidão patológica. Estudos futuros poderão sanar algumas dessas limitações.

\section{CONCLUSÕES}

O presente estudo conseguiu demonstrar que a Escala Brasileira de Solidão UCLA (UCLA-BR) apresenta boas qualidades psicométricas, indicando capacidade para avaliar a solidão de respondentes entre 20 e 87 anos, no contexto brasileiro. O instrumento apresentou evidências de validade de construto e discriminante. Além disso, apresentou bom índice de fidedignidade, indicando ser uma medida confiável. Espera-se que a existência de um instrumento adaptado e validado para a população brasileira contribua para a melhor avaliação emocional desse aspecto em nossa população e aumente o interesse de profissionais de saúde e pesquisadores pela temática.

\section{CONTRIBUIÇÕES INDIVIDUAIS}

Sabrina Martins Barroso - Contribuiu significativamente na concepção do desenho do estudo, bem como na análise e interpretação dos dados, e aprovou a versão final a ser publicada. 
Valéria Sousa de Andrade - Contribuiu substancialmente na revisão crítica do conteúdo intelectual do artigo e aprovou a versão final a ser publicada.

Aida Hutz Midgett - Contribuiu substancialmente na elaboração do artigo.

Rubem Gomes Neves de Carvalho - Contribuiu substancialmente na elaboração do artigo.

\section{CONFLITOS DE INTERESSE}

Os autores não possuem conflitos de interesse a serem declarados.

\section{AGRADECIMENTOS}

Agradecemos à Alessandra Gisloti e à Andressa Pimentel pelo auxílio na coleta de dados deste estudo. Agradecemos à Fundação de Amparo à Pesquisa do Estado de Minas Gerais (Fapemig) pelo apoio financeiro ao projeto sob a forma de bolsa de iniciação científica.

\section{REFERÊNCIAS}

1. Berguno G, Leroux P, McAinsh K, Shaikh S. Children's experience of loneliness at school and its relation to bullying and the quality of teacher interventions. Qual Rep. 2004;9:483-99.

2. Pinquart $M$, Sorensen $S$. Influences on loneliness in older adults: a meta-analysis. BASP. 2001;23:245-66.

3. Sundstrom G, Fransson E, Malmberg B, Davey A. Loneliness among older Europeans. EJA 2009;6:267-75.

4. Hawkley LC, Cacioppo JT. Loneliness matters: a theoretical and empirical review of consequences and mechanisms. Ann Behav Med. 2010;40(2):1-14.

5. Theeke LA. Predictors of loneliness in U.S. adults over age sixty-five. Arch Psychiatr Nurs. 2009;23(5):387-96.

6. Cacioppo JT, Hawley LC, Ernst JM, Burleson M, Berntson GG, Nouriani B, et al. Loneliness with nomological nets: an evolutionary perspective. J Res Pers. 2006;40:1054-85.

7. Levine MP. Loneliness and eating disorders. J Psychol. 2012;146(1-2):243-57.

8. Reichl C, Schneider JF, Spinath FM. Relation of self-talk frequency to loneliness, need to belong, and health in German adults. Pers Individ Dif. 2013;54:241-5.

9. Vanhalst J, Luyckx K, Raes F, Goossens L. Loneliness and depressive symptoms: the mediating and moderating role of uncontrollable ruminative thoughts. J Psychol. 2012;146(12):259-76.

10. Russell D, Peplau LA, Ferguson ML. Developing a measure of loneliness. J Pers Assess. 1978;42(3):290-4.

11. Russell D, Peplau LA, Cutrona CE. The revised UCLA Loneliness Scale: concurrent and discriminant validity evidence. J Pers Soc Psychol. 1980;39(3):472-80.

12. Jaremka LM, Fagundes (P, Peng J, Bennett JM, Glaser R, Malarkey WB, et al. Loneliness promotes inflammation during acute stress. Psychol Sci. 2013;24(7):1089-97.

13. Amieva H, Stoykova R, Matharan F, Helmer C, Antonucci TC, Dartigues JF. What aspects of social network are protective for dementia? Not the quantity but the quality of social interactions is protective up to 15 years later. Psychosom Med. 2010;72(9):905-11.
14. Schinka KC, Van Dulmen MH, Bossarte R, Swahn M. Association between loneliness and suicidality during middle childhood and adolescence: Iongitudinal effects and the role of demographic characteristics. J Psychol. 2012;146(1-2):105-18.

15. Neto F, Barros J. Solidão em diferentes níveis etários. Est Interdis Envelhec. 2001;3:71-88.

16. Fokkema T, De Jong Gierveld J, Dykstra PA. Cross-national differences in older adult loneliness. J Psychol. 2012;146(1-2):201-28.

17. Zhao J, Kong F, Wang Y. The role of social support and self-esteem in the relationship between shyness and loneliness. Pers Individ Dif. 2013;54:577-81.

18. Liepins $M$, Cline T. The development of concepts of loneliness during the early years in school. School Psychol Int. 2011;32(4):397-411.

19. Fernandes H. Solidão em idosos do meio rural do Concelho de Bragança. Disponível em: https://bibliotecadigital.ipb.pt/bitstream/10198/2668/1/Solida0\%20em\%20idosos\%20 do\%20meio\%20rural\%20d0\%20concelho\%20de\%20Braganca.pdf. Acessado em: 12 ag0. 2015.

20. Russell DW, Cutrona CE, MCRae C, Gomez M. Is loneliness the same as being alone? J Psychol. 2012;146(1-2):7-22.

21. Perlman D, Peplau LA. Theoretical approaches to loneliness. In: Peplau LA, Perlman D. Loneliness: a sourcebook of current theory, research, and therapy. New York: Wiley Interscience; 1982.

22. Fontinha MCR. Perspectivas de morte: relação com o suporte social e a solidão em idosos. <http://recil.grupolusofona.pt/bitstream/handle/10437/1293/Tese $\% 20-\% 203 \% 20$ etapa\%201.pdf? sequence=1>. Acessado em: 12 ago. 2015.

23. Oliveira APSV, Silva MM. Fatores que dificultam a perda de peso em mulheres obesas de graus I e II. Rev Psicol Saúde. 2014;6(1):74-82.

24. Marcoen A, Goossens L, Caes P. Lonelines in pre-through late adolescence: exploring the contributions of a multidimensional approach. J Youth Adolesc. 1987;16(6):561-77.

25. Hughes ME, Waite LJ, Hawkley LC, Cacioppo JT. A short scale for measuring loneliness in large surveys: results from two population-based studies. Res Aging. 2004;26(6):655-72.

26. Russel DW. UCLA Loneliness Scale (Version 3): reliability, validity, and factor structure. J Pers Assess. 1996;66(1):20-40.

27. Neto F. Psychometric analysis of the short-form UCLA Loneliness Scale (ULS-6) in older adults. Eur J Ageing. 2014;11:313-9.

28. Doğan T, ÇötokNA, Tekin EG. Reliability and validity of the Turkish Version of the UCLA Loneliness Scale (ULS-8) among university students. Procedia Soc Behav Sci. 2011;15:2058-62.

29. Boffo M, Mannarini S, Munari C. Exploratory structure equation modeling of the UCLA Loneliness Scale: a contribution to the Italian adaptation. TPM. 2012;19(4) Special Issue:345-63.

30. Pinheiro AAA, Tamayo A. Escala UCLA de Solidão: adaptação e validação. Arq Bras Psicol. 1984;36(1):36-44.

31. Conselho Federal de Psicologia. Resolução n0 002/2003 [online]. <http://www.pol.org. br>. Acessado em: 12 ago. 2015.

32. Pasquali L. Instrumentação psicológica: fundamentos e práticas. Porto Alegre: Artmed; 2010.

33. Soares JF, Siqueira AL. Introdução à estatística médica. Belo Horizonte: COOPMED; 2002.

34. Instituto Brasileiro de Geografia e Estatística. Disponível em: <http://www.censo2010. ibge.gov.br/sinopse/index.php?dados=26\&uf=31\#topo_piramide>. Acessado em: 12 ag0. 2015.

35. de Lima Osório F, Vilela Mendes A, Crippa JA, Loureiro SR. Study of the discriminative validity of the PHQ-9 and PHQ-2 in a sample of Brazilian women in the context of primary health care. Perspect Psychiatr Care. 2009;45(3):216-27.

36. Santos IS, Tavares BF, Munhoz TN, Almeida LSP, Silva NTB, Tams BD. Sensibilidade e especificidade do Patient Health Questionnaire-9 (PHQ-9) entre adultos da população geral. Cad Saude Publica. 2013;29(8):1533-43.

37. Siqueira MMM. Construção e validação da escala de percepção de suporte social. Psicol Estud. 2008;13(2):381-8.

38. Ascher SR, Paquette JA. Loneliness and peer relations in childhood. Curr Dir Psychol. 2003;12(3):75-8. 


\section{ANEXO 1}

ESCALA BRASILEIRA DE SOLIDÃO UCLA (UCLA-BR)

Nome:

Data de nascimento: Estado civil:

Sexo: ( ) Feminino( ) Masculino Escolaridade:

INSTRUÇ̃̃ES: Abaixo se encontram várias afirmativas sobre a forma que alguém pode se sentir. Pedimos que leia com atenção e marque com que frequência você se sente como descrito em cada uma das afirmativas abaixo.

\begin{tabular}{|c|c|c|c|c|}
\hline & Nunca & Raramente & Algumas vezes & Frequentemente \\
\hline \multicolumn{5}{|l|}{ Eu me sinto infeliz por fazer tantas coisas sozinho(a). } \\
\hline \multicolumn{5}{|l|}{ Eu não tolero ficar tão sozinho(a). } \\
\hline \multicolumn{5}{|l|}{ Eu sinto que não tenho companhia. } \\
\hline \multicolumn{5}{|l|}{ Eu sinto que ninguém me compreende. } \\
\hline \multicolumn{5}{|l|}{ Eu fico esperando as pessoas me ligarem ou escreverem. } \\
\hline \multicolumn{5}{|l|}{ Eu sinto que não tenho ninguém a quem eu possa recorrer. } \\
\hline \multicolumn{5}{|l|}{ Eu não me sinto próximo(a) a ninguém. } \\
\hline \multicolumn{5}{|c|}{ Sinto que meus interesses e ideias não são compartilhados por aqueles que me rodeiam. } \\
\hline \multicolumn{5}{|l|}{ Eu me sinto excluído(a). } \\
\hline \multicolumn{5}{|l|}{ Eu me sinto completamente sozinho(a). } \\
\hline \multicolumn{5}{|c|}{ Eu sou incapaz de me aproximar e de me comunicar com as pessoas ao meu redor. } \\
\hline \multicolumn{5}{|l|}{ Eu sinto que minhas relações sociais são superficiais. } \\
\hline \multicolumn{5}{|l|}{ Eu me sinto carente de companhia. } \\
\hline \multicolumn{5}{|l|}{ Eu sinto que ninguém me conhece realmente bem. } \\
\hline \multicolumn{5}{|l|}{ Eu me sinto isolado(a) das outras pessoas. } \\
\hline \multicolumn{5}{|l|}{ Sou infeliz estando tão excluído(a). } \\
\hline \multicolumn{5}{|l|}{ Para mim é difícil fazer amigos. } \\
\hline \multicolumn{5}{|l|}{ Eu me sinto bloqueado(a) e excluído(a) por outras pessoas. } \\
\hline \multicolumn{5}{|l|}{ Sinto que as pessoas estão ao meu redor, mas não estão comigo. } \\
\hline Eu me sinto incomodado(a) em realizar atividades sozinho(a). & & & & \\
\hline
\end{tabular}

